\title{
BENEFIT OF TOPIRAMATE IN THE TREATMENT OF SYMPTOMS OF BORDERLINE PERSONALITY DISORDER: A TYPICAL REVIEW
}

\author{
Asheesh Singh $^{1}$, Parul Singh $^{2}$, Chandra Kant Patel $^{3}$ \\ ${ }^{1}$ VNS Institute of Pharmacy, VNS campus, Neelbud, Bhopal, India-462044 \\ ${ }^{2}$ Department of Pharmacy, Guru Ghasidas University, Bilaspur, India-495009 \\ ${ }^{3}$ Sri Satya Sai University of Technology and Medical Science, Sehore, India-466001
}

\begin{abstract}
Topiramate is a new antiepileptic drug (AED) that has been approved worldwide (in more than 80 countries) for the treatment of various kinds of epilepsy. It is currently being evaluated for its effect in various neurological and psychiatric disorders. Topiramate is used alone or with other medications to prevent and control seizures (epilepsy). This medication is also used to prevent migraine headaches and decrease how often you get them. The present review includes all available information like pharmacokinetics, pharmacological action and side effects. Moreover sometimes it is necessary to monitor the therapeutic concentration of Topiramate. The pharmacokinetics of topiramate are characterised by linear pharmacokinetics over the dose range 100-800 mg, low oral clearance $(22-36 \mathrm{~mL} / \mathrm{min})$, which, in immunotherapy, is predominantly through renal excretion (renal clearance $10-20 \mathrm{~mL} / \mathrm{min}$ ), and a long half-life (19-25 hours), which is reduced when co administered with inducing AEDs such as phenytoin, phenobarbital and carbamazepine. This paper provides a critical review of the pharmacokinetic interactions of topiramate with old and new AEDs, an oral contraceptive, and the CNS-active drugs lithium, haloperidol, amitriptyline, risperidone, sumatriptan, propranolol and dihydroergotamine. The results of many of these drug interaction studies with topiramate have not been published before, and are presented and discussed for the first time in this article.
\end{abstract}

Keywords: Antiepileptic drug, bipolar disorder, epilepsy, migraine, obesity, seizures, substance abuse, topiramate, weight loss.

Article Info: Received 22 March 2017; Review Completed 02 April 2017; Accepted 02 April 2017, Available online 15 May 2017 Cite this article as:

Singh A, Singh P, Patel CK, Benefit of Topiramate in the treatment of symptoms of borderline personality disorder: a typical review, Journal of Drug Delivery and Therapeutics. 2017; 7(3):1-7

DOI: http://dx.doi.org/10.22270/jddt.v7i3.1433

*Address for Correspondence

Asheesh Singh, Senior Research Scientist, Department of Analytical and Medicinal Chemistry, VNS Institute of Pharmacy, Neelbud, Bhopal-462044, (B) 9107566553683 |+91-9893737249

Email: asheesh_parihar@yahoo.com

\section{INTRODUCTION}

Topiramate (brand name Topamax) is an anticonvulsant (antiepilepsy) drug. In late 2012, topiramate was approved by the United States Food and Drug Administration (FDA) in combination with phentermine for weight loss. The drug had previously been used offlabel for this purpose ${ }^{1-2}$. Topiramate was originally produced by Ortho-McNeil Neurologics and Noramco, Inc., both divisions of the Johnson \& Johnson Corporation. This medication was discovered in 1979 by Bruce E. Maryanoff and Joseph F. Gardocki during their research work at McNeil Pharmaceutical. Generic versions are available in Canada and were FDA approved in September 2006. Mylan Pharmaceuticals was recently granted final approval for generic topiramate 25, 100, and $200 \mathrm{mg}$ tablets and sprinkle capsules by the FDA for sale in the US. $50 \mathrm{mg}$ tablets were granted tentative approval. The last patent for topiramate in the U.S. was for pediatric use; this patent expired on February 28, 2009.5 On May 21, 2010, Ortho-McNeil plead guilty and was fined US\$6.14 million by the FDA for promoting Topamax to treat psychiatric disorders, without applying for any approval 
and there was no data from any well-controlled clinical trial to demonstrate that Topamax was safe and effective to treat any psychiatric conditions ${ }^{3-5}$.

Topiramate is used to treat epilepsy in children and adults, and it was originally used as an anticonvulsant. In children, it is indicated for the treatment of LennoxGastaut syndrome, a disorder that causes seizures and developmental delay. It is also Food and Drug Administration (FDA) approved for, and most frequently prescribed for, the prevention of migraines. The drug is also used to treat migraines due to the effect it has on the blood vessels in the brain. It has been found to be increasingly effective for migraine sufferers with limited side effects ${ }^{6-7}$.

Psychiatrists have used topiramate to treat bipolar disorder, although the available evidence does not support its use in any phase of bipolar disorder treatment. A more recent review, published in 2010, suggested a benefit of topiramate in the treatment of symptoms of borderline personality disorder, however the authors note that this was based only on one randomized controlled trial and requires replication. Also the authors noted that the long-term effects have not been studied ${ }^{8}$.

Recent clinical reports indicate that it may have mood stabilizing properties. Other off-label and investigational uses of topiramate include the treatment of essential tremor, bulimia nervosa, obsessive-compulsive disorder, idiopathic intracranial hypertension and cluster headache. Topiramate has not been shown to work as a pain medicine in diabetic neuropathy, the only neuropathic condition in which it has been adequately tested. Dispensing errors have been reported between Topamax (topiramate) tablets and Toprol-XL (metoprolol succinate) extended-release tablets thereby requiring extra care to ensure the correct medication is being taken ${ }^{9-12}$.

Half-life: 19 to 23 hours. The mean elimination half-life was 31 hours following repeat administration of the extended-release formulation.

\section{Brand Names}

a. Topamax

b. Trokendi XR

c. Topiragen

d. Qudexy XR

\section{HETEROCYCLIC CHEMISTRY}

Topiramate is a Sulphamate-substituted monosaccharide with a structure distinct from other AEDS. Its chemical formula is $2, \quad 3: 4, \quad 5$-di-O-(1-isopropylidene)- 3 -D fructopyranosesulphamate. It is a derivative

of the naturally-occurring monosaccharide D-fructose. Topiramate has the molecular formula

$\mathrm{C}_{12} \mathrm{H}_{21} \mathrm{NO}_{8} \mathrm{~S}$. It is of Synthetic origin and belongs to Sulphamate Monosaccharides. . The Molecular Weight of Topiramate is $339.40^{13-14}$.

\section{PHARMACEUTICAL ASPECTS}

\section{Solubility}

Topiramate is a white crystalline powder with a bitter taste. Topiramate is most soluble in alkaline solutions containing sodium hydroxide or sodium phosphate and having a $\mathrm{pH}$ of 9 to 10 . It is freely soluble in acetone, chloroform, dimethyl sulfoxide, and ethanol. The solubility in water is $9.8 \mathrm{mg} / \mathrm{mL}$. Its saturated solution has a $\mathrm{pH}$ of 6.3 .

\section{Structure of Topiramate:}

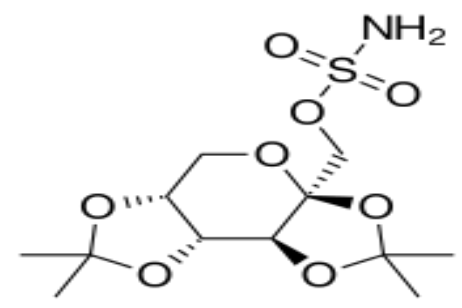

\section{$2,3: 4,5$-Bis- $O$-(1-methylethylidene)- $\beta$-D- fructopyranose sulfamate}

\section{Mechanism of Action}

Topiramate is Soluble in most alkaline solutions containing sodium hydroxide or sodium phosphate and having $\mathrm{pH}$ of $9-10$. It is freely soluble in acetone, chloroform, dimethyl, sulfoxide and ethanol. The precise mechanism of action of topiramate is not known. However, studies have shown that topiramate blocks the action potentials elicited repetitively by a sustained depolarization of the neurons in a time-dependent manner, suggesting a state-dependent sodium channel blocking action. Topiramate also augments the activity of the neurotransmitter gamma-aminobutyrate (GABA) at some subtypes of the GABAA receptor (controls an integral chloride channel), indicating a possible mechanism through potentiation of the activity of GABA $^{15-17}$. Topiramate also demonstrates antagonism of the AMPA/kainate subtype of the glutamate excitatory amino acid receptor. It also inhibits carbonic anhydrase (particularly isozymes II and IV), but this action is weak and unlikely to be related to its anticonvulsant actions ${ }^{18}$.

\section{Topiramate Drug-Drug Interactions}

Topiramate has many drug-drug interactions. Some of the most common are listed below:

- As topiramate inhibits carbonic anhydrase, use with other inhibitors of carbonic anhydrase (e.g. acetazolamide) increases the risk of kidney stones.

- Enzyme inducers (e.g. carbamazepine) can increase the elimination of topiramate, possibly necessitating dose escalations of topiramate.

- Topiramate may increase the plasma-levels of phenytoin.

- $\quad$ Topiramate itself is a weak inhibitor of CYP2C19 and induces CYP3A4; a decrease in plasma levels of estrogens and digoxin has been noted during topiramate therapy. This can reduce the effectiveness of oral contraceptives (birth control pills); use of alternative birth control methods is 
recommended. Neither intrauterine devices (IUDs) nor Depo-Provera are affected by topiramate.

- Alcohol may cause increased sedation or drowsiness, and increase the risk of having a seizure.

- As listed in the 06/29/2005 label posted at the Drugs@FDA website page 14,'conditions or therapies that predispose to acidosis may be additive to the bicarbonate lowering effects of Topiramate.

- Oligohidrosis and hyperthermia were reported in post-marketing reports about topiramate; antimuscarinic drugs (like trospium) can aggravate these disorders ${ }^{19-20}$.

\section{Pharmacology}

Chemically, topiramate is a sulfamate-substituted monosaccharide, related to fructose, a rather unusual chemical structure for an anticonvulsant. Topiramate is quickly absorbed after oral use. Most of the drug (70\%) is excreted in the urine unchanged. The remainder is extensively metabolized by hydroxylation, hydrolysis, and glucuronidation. Six metabolites have been identified in humans, none of which constitutes more than $5 \%$ of an administered dose.

Several cellular targets have been proposed to be relevant to the therapeutic activity of topiramate. These include (1) voltage-gated sodium channels; (2) highvoltage-activated calcium channels; (3) GABA-A receptors; (4) AMPA/kainate receptors; and (5) carbonic anhydrase isoenzymes. There is evidence that topiramate may alter the activity of its targets by modifying their phosphorylation state instead of by a direct action. The effect on sodium channels could be of particular relevance for seizure protection. Although topiramate does inhibit high-voltage-activated calcium channels, the relevance to clinical activity is uncertain. Effects on specific GABA-A receptor isoforms could also contribute to the antiseizure activity of the drug. Topiramate selectively inhibits cytosolic (type II) and membrane associated (type IV) forms of carbonic anhydrase. The action on carbonic anhydrase isoenzymes may contribute to the drug's side-effects, including its propensity to cause metabolic acidosis and calcium phosphate kidney stones. Topiramate inhibits maximal electroshock and pentylenetetrazol-induced seizures as well as partial and secondarily generalized tonic-clonic seizures in the kindling model, findings predictive of a broad spectrum of activities clinically. Its action on mitochondrial permeability transition pores has been proposed as a mechanism ${ }^{21-22}$.

While many anticonvulsants have been associated with apoptosis in young animals, animal experiments have found that topiramate is the only anticonvulsant that does not induce apoptosis in young animals at doses needed to produce an anticonvulsant effect ${ }^{23}$.

\section{PHARMACOKINETICS AND METABOLISM OF TOPIRAMATE}

The pharmacokinetics and pharmacokinetic interactions of topiramate (TPM) in humans have been studied quite extensively. Topiramate is rapidly absorbed, with a high bioavailability that is not affected by concomitant food intake. The volume of distribution is $0.6-0.8 \mathrm{1} / \mathrm{kg}$, suggesting distribution into total body water. The binding of TPM to serum proteins is low, which precludes the displacement interactions that are seen between highly bound drugs such as valproate and phenytoin. The elimination kinetics of TPM are strictly linear and, accordingly, there is a linear relationship between maintenance dose and steady-state plasma levels. Topiramate is excreted predominantly by the kidneys as unmetabolized drug. This is generally associated with lower interpatient variability in elimination kinetics. Approximately $20 \%$ of orally administered TPM is metabolized in the liver and this fraction may increase up to $50 \%$ in the presence of enzyme-inducing drugs, such as phenytoin or carbamazepine. During chronic ingestion of TPM, there is no clinically significant accumulation of any active metabolite, even in patients taking enzyme-inducing drugs. The elimination half-life of TPM is relatively long and does not require more frequent than twice-daily dosing. Finally, TPM has a relatively low potential for drug interactions. The clinically significant pharmacokinetic interactions between TPM and other antiepileptic drugs are limited to an increase in the clearance of TPM when inducing drugs such as phenytoin or carbamazepine are added. TPM has little or no effect on the pharmacokinetics of other antiepileptic drugs, but it can increase the clearance of the estrogenic component of oral contraceptives by up to $30 \%{ }^{24-25}$.

The absolute bioavailability, or oral availability, of topiramate is $81-95 \%$ and is not affected by food. Although topiramate is not extensively metabolised when administered in monotherapy (fraction metabolised approximately 20\%), its metabolism is induced during polytherapy with carbamazepine and phenytoin, and, consequently, its fraction metabolised increases. During concomitant treatment with topiramate and carbamazepine or phenytoin, the (oral) clearance of topiramate increases 2-fold and its half-life becomes shorter by approximately 50\%, which may require topiramate dosage adjustment when phenytoin or carbamazepine therapy is added or discontinued. From a pharmacokinetic standpoint, topiramate is a unique example of a drug that, because of its major renal elimination component, is not subject to drug interaction due to enzyme inhibition, but nevertheless is susceptible to clinically relevant drug interactions due to induction of its metabolism. Unlike old AEDs such as phenytoin and carbamazepine, topiramate is a mild inducer and, currently, the only interaction observed as a result of induction by topiramate is that with ethinylestradiol. Topiramate only increases the oral clearance of ethinylestradiol in an oral contraceptive at high dosages (>200 mg/day). Because of this dose-dependency, possible interactions between topiramate and oral contraceptives should be assessed according to the topiramate dosage utilized ${ }^{26}$.

\section{POSSIBLE SIDE EFFECTS OF TOPIRAMATE:}

All medicines may cause side effects, but many people have no, or minor, side effects. Check with your doctor 
if any of these most COMMON side effects persist or become bothersome:

Constipation; diarrhea; dizziness; drowsiness; dry mouth; flushing; headache; loss of appetite; nausea; nervousness; stomach pain or upset; symptoms of upper respiratory tract infection (eg, cough, mild sore throat, running or stuffy nose, sneezing); taste changes; tiredness; trouble sleeping; weakness; weight loss.

\section{Nervous system:}

The majorities of cognitive-related adverse events were mild to moderate in severity and frequently occurred in isolation. Rapid titration rate and higher initial doses were associated with higher incidences of cognitiverelated adverse events.

Very common (10\% or more): Paresthesia, somnolence, dizziness.

Common (1\% to 10\%): Disturbance in attention, memory impairment, amnesia, cognitive disorder, mental impairment, psychomotor skills impaired, convulsion, coordination abnormal, tremor, lethargy, hypothesia, nystagmus, dysgeusia, balance disorder, dysarthria, intention tremor, sedation

Uncommon $(0.1 \%$ to $1 \%)$ : Depressed level of consciousness, grand mal convulsion, visual field defect, complex partial seizures, speech disorder, psychomotor hyperactivity, syncope, sensory disturbance, drooling, hypersomnia, aphasia, repetitive speech, hypokinesia, dyskinesia, dizziness postural, poor quality sleep, burning sensation, sensory loss, parosmia, cerebellar syndrome, dysesthesia, hypogeusia, stupor, clumsiness, aura, ageusia, dysgraphia, dysphasia, neuropathy peripheral, presyncope, dystonia, formication

Rare (less than 0.1\%): Apraxia, circadian rhythm sleep disorder, hyperesthesia, hyposmia, anosmia, essential tremor, akinesia, unresponsive to stimuli.

Hepatic: Rare (less than 0.1\%): Hepatitis, hepatic failure, increase in liver enzymes

Ocular: The primary treatment of cases of secondary angle closure glaucoma cause by topiramate is discontinuation of the drug.

Common (1\% to 10\%): Vision blurred, diplopia, visual disturbance

Uncommon (0.1\% to $1 \%)$ : Visual acuity reduced, scotoma, myopia, abnormal sensation in eye, dry eye, photophobia, blepharospasm, lacrimation increased, photopsia, mydriasis, presbyopia

Rare (less than 0.1\%): Blindness unilateral, blindness transient, glaucoma, accommodation disorder, altered visual depth perception, scintillating scotoma, eyelid edema, night blindness, amblyopia

Very rare (less than 0.01\%): Frequency not reported: Angle closure glaucoma, maculopathy, eye movement disorder.

\section{Gastrointestinal:}

Very common (10\% or more): Nausea, diarrhea
Common (1\% to 10\%): Vomiting, constipation, abdominal pain upper, dyspepsia, abdominal pain, dry mouth, stomach discomfort, paresthesia oral, gastritis, abdominal discomfort

Uncommon (0.1\% to 1\%): Pancreatitis, flatulence, gastroesophageal reflux disease, abdominal pain lower, hypoesthesia oral, gingival bleeding, abdominal distension, epigastric discomfort, abdominal tenderness, salivary hypersecretion, oral pain, breath odor, glossodynia.

Cardiovascular: Uncommon $(0.1 \%$ to $1 \%)$ : Bradycardia, sinus bradycardia, palpitations, hypotension, orthostatic hypotension, flushing, hot flushes Rare (less than $0.1 \%$ ): Raynaud's phenomenon.

\section{Hematologic:}

Common (1\% to $10 \%)$ : Anemia, epistaxis

Uncommon $\quad(0.1 \%$ to $1 \%)$ : Leucopenia, thrombocytopenia lymphadenopathy, white blood cell count decreased eosinophilia.

Rare (less than 0.1\%): Neutropenia.

Metabolic: Very common (10\% or more): Weight decreased

Common (1\% to 10\%): Anorexia, decreased appetite, weight increased.

Uncommon (0.1\% to 1\%): Metabolic acidosis, hypokalemia, increased appetite, polydipsia

Rare (less than 0.1\%): Acidosis hyperchloremic, blood bicarbonate decreased.

\section{Musculoskeletal:}

Common (1\% to 10\%): Arthralgia, muscle spasms, myalgia, muscle twitching, muscular weakness, musculoskeletal chest pain

Uncommon $(0.1 \%$ to $1 \%)$ : Joint swelling, musculoskeletal stiffness, flank pain, muscle fatigue

Rare (less than 0.1\%): Limb discomfort.

Psychiatric: Depression and mood problems were dose related.

Psychiatric side effects including insomnia, personality disorder, impotence, hallucination, euphoria, psychosis, decreased libido, anxiety, depression, mood problems, and suicide attempts have been reported. Two cases of panic attacks have also been reported.

Genitourinary: Uncommon $(0.1 \%$ to $1 \%)$ : Erectile dysfunction, sexual dysfunction, crystal urine present, intermenstrual bleeding, leucorrhea, menorrhagia, vaginitis, and amenorrhea., urinary tract infections, micturition frequency, urinary incontinence, dysuria.

Respiratory: Respiratory side effects including pulmonary embolism, coughing, pneumonia, asthma, and bronchitis have been reported.

\section{Dermatologic:}

Common (1\% to 10\%): Alopecia, rash, pruritus 
Uncommon (0.1\% to 1\%): Anhidrosis, hypoesthesia facial, urticaria, erythema, pruritus generalized, rash macular, skin discoloration, dermatitis allergic, swelling face.

Rare (less than 0.1\%): Stevens-Johnson syndrome, erythema multiforme, skin odor abnormal, periorbital edema, urticaria localized.

Others: Very common (10\% or more): Fatigue

Renal: Renal side effects including kidney stones $(1.3 \%$ to $1.5 \%$ ) and renal tubular acidosis have been reported.

Hypersensitivity: Common (1\% to 10\%): Hypersensitivity Frequency not reported: Allergic edema, conjunctival edema ${ }^{27-28}$.

\section{IMPORTANT SAFETY INFORMATION ABOUT TOPIRAMATE}

a) Topiramate may cause drowsiness, dizziness, confusion, trouble concentrating, or vision changes. These effects may be worse if you take it with alcohol or certain medicines. Use topiramate with caution. Do not drive or perform other possibly unsafe tasks until you know how you react to it.

b) Do not change your dose without checking with your doctor.

c) Do not drink alcohol while you take topiramate.

d) Check with your doctor before you use medicines that may cause drowsiness (eg, sleep aids, muscle relaxers) while you are taking topiramate; it may add to their effects. Ask your pharmacist if you have questions about which medicines may cause drowsiness.

e) Topiramate may reduce sweating, which could raise body temperature, especially in children. This risk is greater in hot weather and/or during vigorous exercise. Drink plenty of fluids. Dress lightly in hot climates or when exercising. Check carefully for signs of decreased sweating. If this occurs, promptly seek cooler or air-conditioned shelter and/or stop exercising. Seek medical attention right away if you have decreased sweating, fever, mental or mood changes, headache, or dizziness.

f) Topiramate may cause high blood acid levels (metabolic acidosis). The risk may be greater in children and in patients with kidney problems, severe breathing problems, or diarrhea. It may also be greater in patients who are taking certain other medicines (eg, acetazolamide), will be having surgery, or are on a ketogenic diet. Over a period of time, metabolic acidosis may cause kidney stones, bone problems, or decreased growth in children. Contact your doctor immediately if you experience fast breathing, unusual tiredness or weakness, sluggishness, persistent loss of appetite, or fast or irregular heartbeat.

g) Topiramate may cause high blood ammonia levels (hyperammonemia). This risk may be greater in patients with metabolism problems or certain liver problems. It may also be greater in patients who are taking valproic acid. Contact your doctor immediately if you experience decreased alertness, mental changes, sluggishness, unusual tiredness, or vomiting.

h) Patients who take topiramate may be at increased risk of suicidal thoughts or actions. The risk may be greater in patients who have had suicidal thoughts or actions in the past. Watch patients who take topiramate closely. Contact the doctor at once if new, worsened, or sudden symptoms such as depressed mood; anxious, restless, or irritable behavior; panic attacks; or any unusual change in mood or behavior occur. Contact the doctor right away if any signs of suicidal thoughts or actions occur.

i) Topiramate may cause serious eye problems that could lead to permanent loss of vision if not treated. Seek medical attention right away if you experience new eye symptoms (eg, blurred vision or other vision changes, eye pain or redness).

j) Caution is advised when using topiramate in Children; they may be more sensitive to its effects, especially decreased sweating and decreased bicarbonate levels.

k) Topiramate may affect growth rate in Children and teenagers in some cases. They may need regular growth checks while they take topiramate.

1) Topiramate may cause birth defects if you take it while you are pregnant. If you may become pregnant, discuss other possible treatment options with your doctor. If a decision is made to take topiramate, use effective birth control while you are taking it. Talk with your doctor about the best kind of birth control to use while taking topiramate, if you are planning to become pregnant, and if you have questions or concerns about this information.

m) Pregnancy and Breast-feeding: Topiramate may cause birth defects if you take it while you are pregnant. If you think you may be pregnant, contact your doctor right away. You will need to discuss the benefits and risks of taking topiramate while you are pregnant. You and your doctor will need to decide if you will continue to take topiramate while you are pregnant. Topiramate is found in breast milk. If you are or will be breast-feeding while you take topiramate, check with your doctor. Discuss any possible risks to your baby ${ }^{29-30}$.

\section{CONCLUSION WITH FUTURE PROSPECTIVE:}

Great progress has been made in the last 150 years in the pharmacological management of epilepsy, and, despite the increasing number of technological advances available, antiepileptic drugs (AEDs) remain the mainstay of treatment for the vast majority of patients with epilepsy. The strengths and weaknesses of those AEDs which are currently licensed are examined, and ways in which their use may be improved are discussed (e.g. rational combinations, use of new formulations). Potentially new targets that may allow the development of effective treatments are highlighted (neuroimmunological manipulation, decreasing inherent drug resistance mechanisms, and modification of adenosine neurotransmission), and a summary of the 
most promising AEDs currently in development is provide.

Among some 14 new antiepileptic drugs (AEDs), those most extensively tested in humans include topiramate (TPM), gabapentin (GBP), lamotrigine (LTG), oxcarbazepine (OCBZ), vigabatrin (VGB), and zonisamide (ZNS). All are currently marketed in some but not all countries. Although no large, comparative studies on efficacy have been conducted, all of these new AEDs are effective in adult localization-related epilepsies, and some have activity in specific syndromes. Although these drugs all have some CNS side effects, especially when administered in combination with other AEDs, they also all have low toxicity profiles.

Currently information is available for 20 potential antiseizure drugs in clinical development. They include candidates with mechanisms of action similar to those of marketed AEDs (allopregnanolone, brivaracetam,

\section{REFERENCES:}

1. Montcriol A, Meaudre E, Kenane N, Asencio Y, Bordes J, Palmier B. Hyperventilation and cerebrospi-nal fluid acidosis caused by topiramate. Ann Pharmacother 42 2008; 42: 58473.

2. Shorvon SD. Safety of topiramate: adverse events and relationships to dosing. Epilepsia. 1996.

3. Newman LC, Broner SW, Lay CL. Reversible anorgasmia with topiramate therapy for migraine. Neurology 2005; 65: 1333-4.

4. Bjoro K, Gjerstad L, Bentdal O, Osnes S, Schrumpf E. Topiramate and fulminant liver failure. Lancet 1998; 352: 11-19.

5. Westergren T, Hjelmeland K, Kristoffersen B. Probable topiramate-induced diarrhea in a 2-month-old breast-fed child - A case report. Epilepsy Behav Case Report. 2014; 2: 22-3.

6. Ohman I, Luef G, Tomson T. Topiramate kinetics during lactation. Epilep 2007; 48:156-7.

7. Marcotte D. Use of topiramate, a new anti-epileptic as a mood stabilizer. J Affect Disord 1998; 50:245-251.

8. Sabers A, Gram L. Drug treatment of epilepsy in the 1990s: Achievements and new developments. Drugs 1996; 52:483493.

9. Ghaemi S, Goodwin F. Use of atypical antipsychotic agents in bipolar and schizoaffective disorders: review of the empirical literature. J Clin Psychopharmacol 1999; 19:354361.

10. Calabrese JR, Keck PE, Jr, McElroy SL, Shelton MD. A pilot study of topiramate as mono therapy in the treatment of acute mania. J Clin Psychopharmacol 2001; 21:340-342.

11. Chengappa KN, Rathore D, Levine J, Atzert R, Solai L, Parepally $\mathrm{H}$, et al. Topiramate as add on treatment for patients with bipolar mania. Bipolar Disord 1999; 1:42-53.

12. Grunze HC, Normann C, Langosch J, Schaefer M, Amann B, Sterr A, et al. Antimanic efficacy of topiramate in 11 patients in an open trial with an on-off-on design. J Clin Psychiatry 2001; 62:464-468.

13. McElroy SL, Suppes T, Keck PE, Frye MA, Denicoff KD, Altshuler LL, et al. Open-label adjunctive topiramate in the treatment of bipolar disorders. Biol Psychiatry 2000; 47:1025-1033.

14. Vieta E, Gilabert A, Rodriguez A, Garcia-Castrillon A, Luna MJ, Arrufat E, et al. Effectiveness and safety of topiramate in treatment-resistant bipolar disorder. Actas Espanolas de Psiquiatria 2001; 29:148-152

15. McIntyre R, Mancini D, McCann S, Srinivasan J, Kennedy S. Topiramate vs. bupropion SR added to mood stabilizer therapy for the depressive phase of bipolar disorder: A single blind study (abstract). Annual meeting of the Society of ganaxolone, ICA-105665, NS1209, selurampanel); those with new mechanisms of action (beprodon, VX-765); compounds repurposed for the treatment of epilepsy (biperiden, bumetanide, fenfluramine, melatonin, nalutozan, pitolisant, quinidine, valnoctamide, verapamil). Clinical development of anti-seizure drugs is still active but unexciting. Potential anti-seizure drugs continue to be largely identified by their activity against seizures provoked by electrical or chemical procedures in animals with normal brains. As in the past, this may lead to new drugs whose efficacy is not better than that of those already on the market ${ }^{31-32}$.

\section{ACKNOWLEDGEMENTS:}

Authors are thankful to VNS Institute of Pharmacy, Bhopal and Department of Pharmacy, GGU Bilaspur for providing the necessary facilities \& guidance to complete this review.

Neuropsychoendocrinology, Rhodes, Greece, 2000; 16:1118 .

16. Eads LA, Kramer T, Wooten G. Effects of topiramate on global functioning in treatment-refractory mood disorders (abstract). 22nd Congress of the Collegium International Psychopharmacologicum, Brussels, Belgium, 2000.

17. Vieta E, Gilabert A, Rodriguez A, Garcia-Castrillon A, Luna MJ, Arrufat F, et al. Topiramate in the adjunctive treatment of refractory bipolar disorder. 22nd Congress of the Collegium Internationale Psychopharmacologicum, Brussels, Belgium, 2010.

18. Sachs GS, Demopulos C, Ghaemi SN, Sambur M, Cosgrove $\mathrm{V}$, Gaughan S, et al. Topiramate in the treatment of refractory bipolar mood disorder. 22nd Congress of the Collegium Internationale Psychopharmacologicum, Brussels, Belgium, 2010.

19. Plon L, Maguire G, Singh P. Topiramate in the treatment of refractory bipolar disorder. Ninth Congress of the International Psychogeriatric Association, Vancouver, Canada, August 1999.

20. Kusumakar V, Yatham L, Kutcher S, O'Donovan C. Use of topiramate in rapid-cycling bipolar women. Ninth Congress of the International Psychogeriatric Association, Vancouver, Canada, 1999.

21. Hahn MK, Cohn T, Teo C, Remington G. Topiramate in schizophrenia: a review of effects on psychopathology and metabolic parameters. Clinical schizophrenia \& related psychoses 2013; 4: 186-96.

22. Lainez MJ, Pascual J, Pascual AM, Santonja JM, Ponz A, Salvador. Topiramate in the prophylactic treatment of cluster headache. Headache 2013; 43: 784-9.

23. Hunt S, Russell A, Smithson WH, Parsons L, Robertson I, Waddell RJ. Topiramate in pregnancy: preliminary experience from the UK Epilepsy and Pregnancy Register". Neuro 2014; 71: 272-6.

24. Kashyap R, Subrahmanyam EVS, Sharbaraya AR. Development and validation of new colorimetric method for the estimation of topiramate in bulk and dosage form. Inter $\mathrm{j}$ of pharm and tech 2012; 4: 3974-3989.

25. Brandt C, Elsner H, Furatsch N. Topiramate overdose: a case report of a patient with extremely high topiramate serum concentrations and nonconvulsive status epilepticus. Epilep 201551: 1090-1093.

26. Mirza Nasir, Marson Anthony G, Pirmohamed Munir. Effect of topiramate on acid-base balance: extent, mechanism and effects. British Journal of Clinical Pharmacology 2012, 68: $655-61$. 
27. Kashyap R, Subrahmanyam EVS \& Sharbaraya AR. Review on Topiramate and Available Analytical Methods. Inter J of Pharma and Chemical Sci 2012; 2:877-883.

28. William RG. Clinical Pharmacology of Topiramate: A Review Lippincott Williams \& Wilkins, Philadelphia International League Against Epilepsy Epilepsiu 2000; 41:S61-S65

29. Gisclon LG, Curtin CR. The pharmacokinetics (PK) of topiramate $(\mathrm{T})$ in subjects with end-stage renal disease undergoing hemodialysis. Clin Pharmacol Ther 1994; 55:196-203.
30. Doose DR, Walker SA, Venkataramanan R, Rabinovitz M, Lever J. Topiramate pharmacokinetics in subjects with liver impairment. Pharm Res 1994; 1 1: S446.

31. Doose DR, Walker SA, Baldassarre J. The effect of food on the oral bioavailability of topiramate from an investigational pediatric sprinkle formulation. Epilepsiu 1997; 38: 147-153.

32. Gaetano Zaccara, Dieter Schmidt. Do traditional anti-seizure drugs have a future? A review of potential anti-seizure drugs in clinical development. Pharma Res 2016; 104:38-48. 